\title{
Research Article \\ Effects of Upregulation of TNFAIP3 on Diabetic Neuropathic Pain in Mice
}

\author{
Yang Liu, ${ }^{1}$ Jinhe Li, ${ }^{1}$ Hongbo Yao, ${ }^{2}$ Meng Zhang, ${ }^{2}$ Jie Lian, ${ }^{2}$ Haiyan Zhang, \\ Keshuang Zhang, ${ }^{2}$ Danyang Liu, ${ }^{2}$ Jiwei Chen, ${ }^{2}$ Yuejing Wang $\mathbb{D}^{2},{ }^{2}$ and Yin Gao $\mathbb{i D}^{3}$ \\ ${ }^{1}$ Department of General Surgery, Qiqihar First Hospital, Qiqihar 161006, China \\ ${ }^{2}$ Department of Embryology, Qiqihar Medical College, Qiqihar 161006, China \\ ${ }^{3}$ Department of Anatomy, Qiqihar Medical College, Qiqihar 161006, China
}

Correspondence should be addressed to Yuejing Wang; 335187646@qq.com and Yin Gao; 2597610698@qq.com

Received 8 September 2021; Accepted 18 October 2021; Published 22 November 2021

Academic Editor: Yuzhen Xu

Copyright (c) 2021 Yang Liu et al. This is an open access article distributed under the Creative Commons Attribution License, which permits unrestricted use, distribution, and reproduction in any medium, provided the original work is properly cited.

\begin{abstract}
Globally, diabetes has assumed epidemic proportions with the neuropathic complications attributed to the malady emerging as a substantial burden on patients and society. DNP has greatly affected the daily life of patients, the effect of traditional treatment methods is not ideal, and it is easy to produce drug resistance. This work is aimed at scrutinizing the effect of upregulating the expression of TNFAIP3 on diabetic neuralgia in mice. This work entailed ascertaining the effects of TNFAIP3 on a murine DNP system. This inspired us to observe the analgesic effect via high expression of lentivirusmediated TNFAIP3 by intrathecal injection in the animal model to explore its regulatory impacts, symptom relief, and mechanistic role in pain. The results displayed an attenuation of hind paw pain hypersensitivity by LV-TNFAIP3 in the animals. The spinal cord and dorsal root ganglion of mice with neuropathic pain displayed an evident dip in TNFAIP3. Inhibition of the ERK/NF- $\kappa \mathrm{B}$ signaling pathway employing LV-TNFAIP3 conspicuously suppressed this pathway while the diabetic pain hypersensitivity was quelled. This effect was also seen with insulin treatment evidently. In conclusion, according to the above analyses, the interaction between DNP and extracellular signal-regulated kinase signal transduction pathway is one of the key factors of pathogenesis.
\end{abstract}

\section{Introduction}

Globally, diabetes has assumed epidemic proportions with the neuropathic complications attributed to the malady emerging as a substantial burden on patients and society. Neuropathic pain is caused by diseases of the body. When neuropathic pain is diagnosed, damage to the sensory nervous system is seen with DNP impacting up to $8 \%$ of the population [1]. The problem of DNP is its severity with effective treatments yet to see the light of the day. Diabetes is mainly divided into two types: type 1 diabetes and type 2 diabetes. DNP is a ubiquitous complication of diabetes. The lifetime incidence of DNP in type 2 diabetics is $45 \sim 70 \%[2,3]$. The early symptom of DNP is sensory nerve disorder with patients usually experiencing sensory symptoms, such as pain, tingling, paresthesia, and numbness $[4,5]$.

DNP has greatly affected the daily life of patients, the effect of traditional treatment methods is not ideal, and it is easy to produce drug resistance $[6,7]$. Therefore, in this study, gene therapy is selected to alleviate the pain. One of the key problems of gene therapy is to select the appropriate vector to make the treatment gene safely and efficiently transferred into the target cells. This work is aimed at scrutinizing the effect of upregulating the expression of TNFAIP3 on diabetic neuralgia in mice. This entails an intrathecal injection of lentiviral-based TNFAIP3 to 
upregulate the expression of TNFAIP3 in a DNP mouse model along with the involvement of the ERK/NF- $\kappa \mathrm{B}$ signaling pathway. Further, pain in the spinal cord and dorsal root ganglia (DRGs) was also explored.

\section{Research Design and Methods}

2.1. Murine DNP Model Generation. All experiments were approved by the Animal Protection and Use Committee of Qiqihar Medical University. C57/BL6 mice (5-7-week-old: $16-25 \mathrm{~g}$ in weight) were housed in cages (4/cage) in a 12 hour-12 hour light-dark cycle at $25 \pm 1^{\circ} \mathrm{C}$. The mice were allowed to drink tap water and eat standard laboratory food at will. Following a 28-day high-sugar and high-fat feed, assays were initiated. Fasting ensued (inclusive of water) for more than 12 hours. Diabetes induction entailed the intraperitoneal injection of $30 \mathrm{mg} / \mathrm{kg}$ streptozotocin (STZ), a total of 2 times, 3 days apart that was ascertained by a fasting blood glucose level $>11.1 \mathrm{~mol} / \mathrm{L}$. This was followed by an instantaneous screening of mice manifesting pain complications that were indicative of the successfully establishing the DNP model. Screened DNP models were further used in the study.

2.2. Lentiviral Vector Intrathecal Injection. Following an intraperitoneal injection with $10 \%$ chloral hydrate to anesthetize an animal, the L4-6 lumbar spinous process was secured by one hand, and the skin pierced with a thick needle to pierce the subarachnoid with a 2-5ul micro syringe employing the other hand. Following penetration of the cavity, the needle was inserted until the typical rat tail quivers and shakes indicative of successful puncture and intrathecal Lv-TNFAIP3 injection.

2.3. Measurement of Hind Paw Withdrawal Threshold. Prior to commencing the ascertaining of this threshold, placing the animals for one week in the test environment was done to facilitate habituation to the surrounding. Following the oneweek habituation, the experiment entailed two approaches: paw withdrawal threshold (PWT) and thermal paw withdrawal latency (PWL). The former is the response to the Von Frey filament stimulation based on radiant heat application to the plantar surface of hind paw while the latter is in response to the Von Frey filament stimulation. All experiments were on the lines of earlier research [8]. All behavioral experiments were started employing blinded conditions.

2.4. Western Blotting Analysis. This was done to quantify TNFAIP3, ERK2, ERK1/2, MAPKp38, NF-KB, and p65 levels in L4-L6 of the spinal cord and DRGs [9]. The DPN mice and controls were the sources of tissues with the former entailing with or without insulin treatment-based processing. Following LV-TNFAIP3 treatment and siRNA treatment, TNFAIP3 (1:500, Abcam, USA) and antibodies against ERK2, ERK1/2, MAPKp38, NF-KB, p65 antibodies (1:800, Abcam, USA), and corresponding horseradish peroxidase-conjugated secondary anti-rat antibodies (at $1: 1000$ dilution) were employed for the assay.
2.5. Toluidine Blue Staining and Immunohistochemical Staining. This entailed the following: once the animals were anesthetized, 4\% paraformaldehyde-based perfusion was done followed by sampling. The spinal cord and DRGs were embedded in paraffin and sliced as 10 micrometers thick sections employing a paraffin microtome. Subsequent toluidine blue staining entailed the use of the staining kit adhering to the prescribed protocols. Immunohistochemistry employed the use of TNFAIP3 (Rabbit, 1:200, Abcam, USA) followed by assessing and imaging in a confocal microscope.

2.6. Data Analysis. This entailed the use of SPSS statistics 17.0 for results expressed as the mean \pm SEM. Two sample $t$-test was employed for significance determination. Application ImageJ software analysis (NIH, Bethesda, Maryland, USA) was employed for Western blot results for both intraand intergroup analyses. Significance across groups was at a $P$ value less than 0.05 .

\section{Results}

All results are as shown in Figures 1-4.

In Figure 1, the results of Western blot were shown in (a). (b)-(f) are the analyses. (b) shows conspicuously lower TNFAIP3 level in DNP vs. sham. (c) and (d) show the conspicuous increase in ERK2 and MAPK proteins, respectively, in DNP vs. sham. Evidently higher NF- $\kappa \mathrm{B}$ in DNP vs. sham. (g) and (k) show the mechanical pain threshold and thermal pain threshold. The pain threshold in the DNP group was significantly decreased at the 2nd, 4 th, 6th, and 8 th week vs. sham $(P<0.05$ for all tests).

In Figure 2, results of toluidine blue staining of mouse ganglion tissue are shown in (a)-(c).

(a) is Con group, (b) is the DNP siRNA group, and (c) is the DNP-TNFAIP3 group. The contour of ganglion neurons of the DNPsiRNA group is not clear vs. sham: Nissl body is not clear, the nucleolus is not obvious, and cell survival rate is low. However, the sham and DNP-TNFAIP3 groups display a clear contour and Nissl body with an obvious nucleolus.

Immunohistochemical staining results of mouse ganglion tissues are shown in (d)-(f), (d) is sham group, (e) is the DNPsiRNA group, (f) is the DNP-TNFAIP3 group, and $(\mathrm{g})$ is gray value analysis of each group. Lower TNFAIP3 in the DNP siRNA set vs. sham while the DNP-TNFAIP3 group displayed an evident increase in TNFAIP3 (* and ${ }^{\#} P<0.05$ ).

In Figure 3, the results of toluidine blue staining of mouse spinal cord tissue are shown below.

(a) $(20 \mu \mathrm{m})$ is sham, (b) $(20 \mu \mathrm{m})$ is the DNP siRNA group, and (c) $(20 \mu \mathrm{m})$ is the DNP-TNFAIP3 group. Compared with sham, the outlines of ganglion neurons, Nissl body, and nucleolus in the DNP siRNA group are not clear; however, they are clear in sham and DNP-TNFAIP3 groups.

Immunohistochemical staining results of spinal cord tissue of mice are shown in (d)-(f).

(d) $(20 \mu \mathrm{m})$ is sham, (e) $(20 \mu \mathrm{m})$ is the DNP siRNA group, (f) $(20 \mu \mathrm{m})$ is the DNP-TNFAIP3 group, and (g) is the gray value analysis of each group. Lowered TNFAIP3 


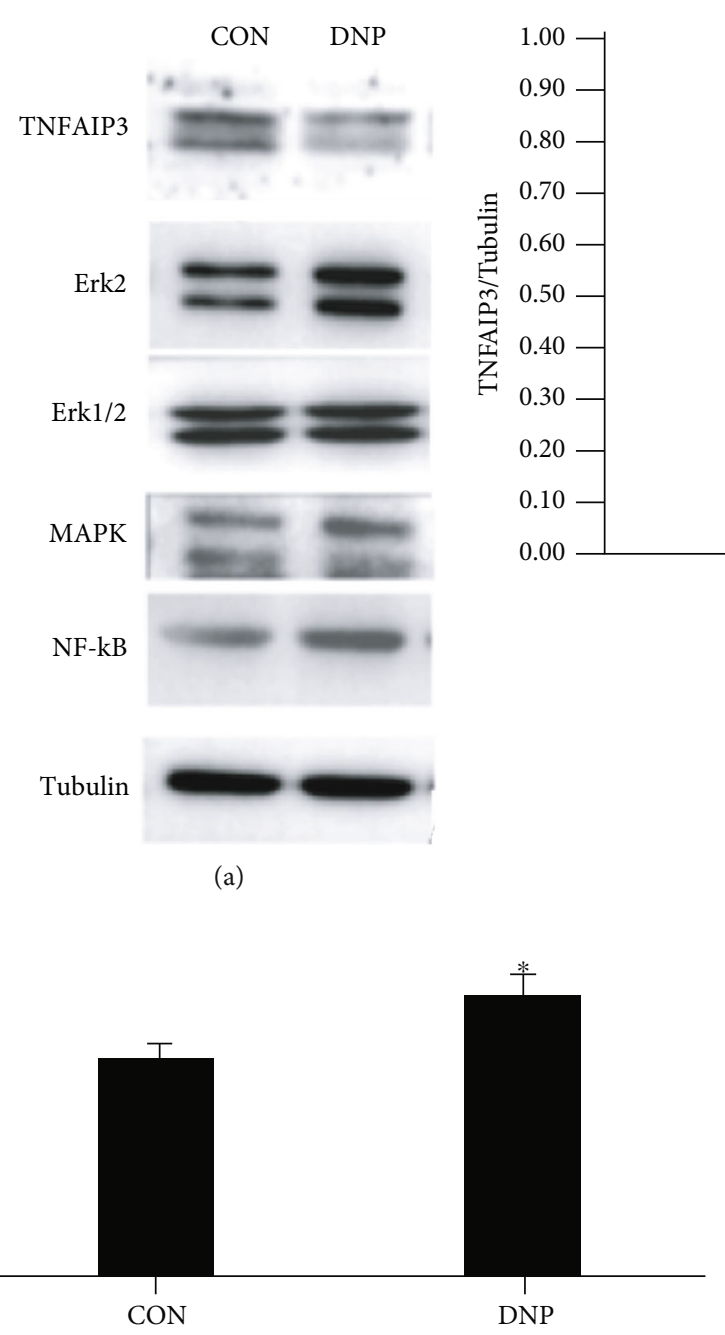

(c)

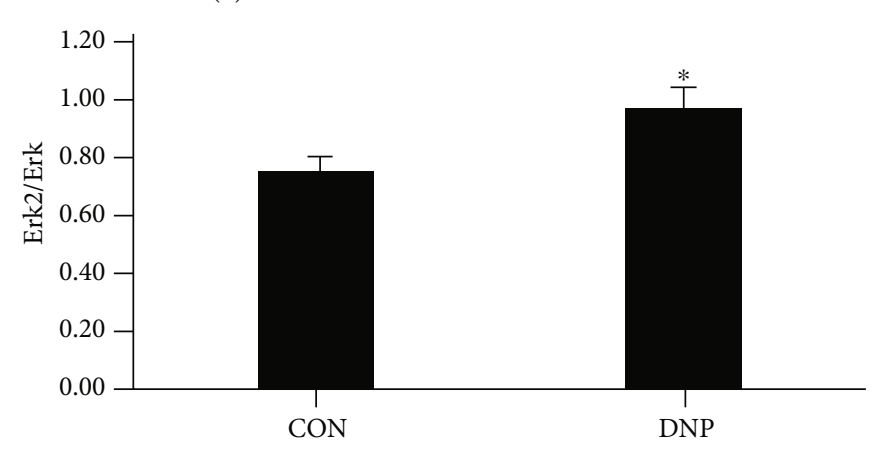

(d)

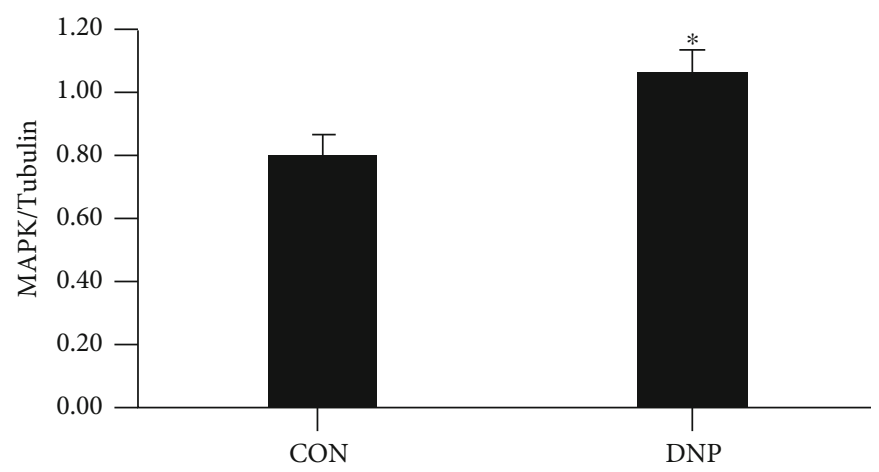

(e)

Figure 1: Continued. 


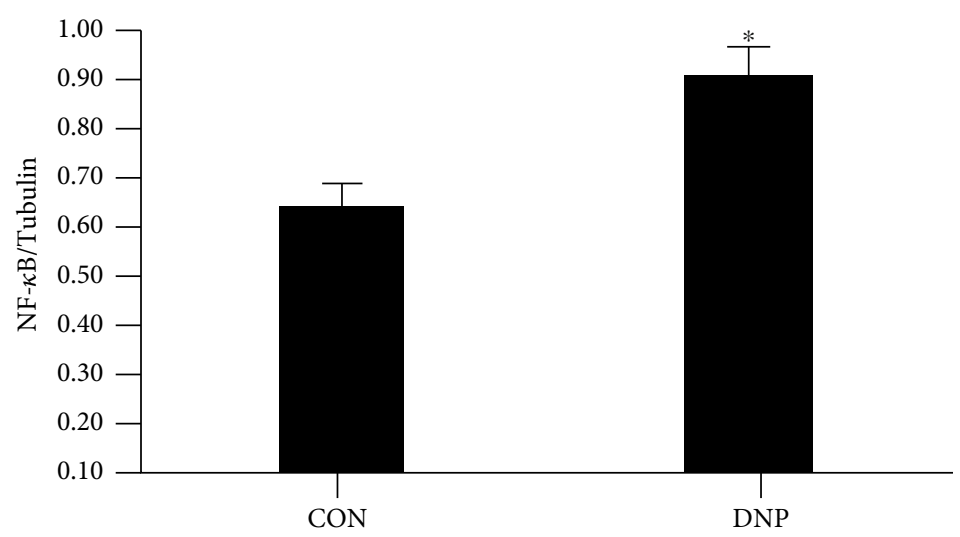

(f)

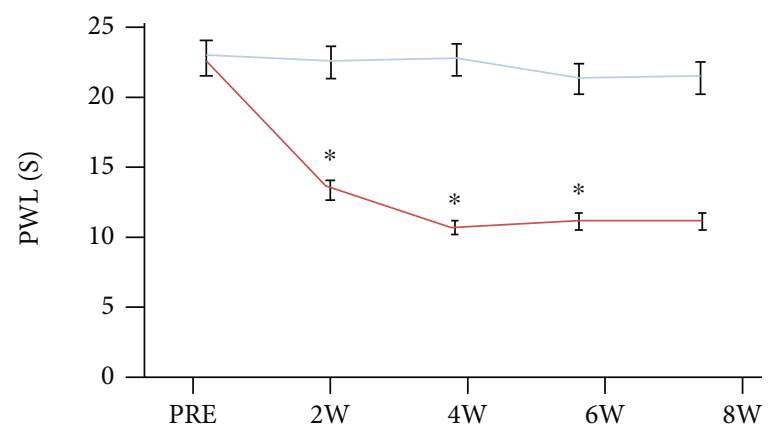

(g)

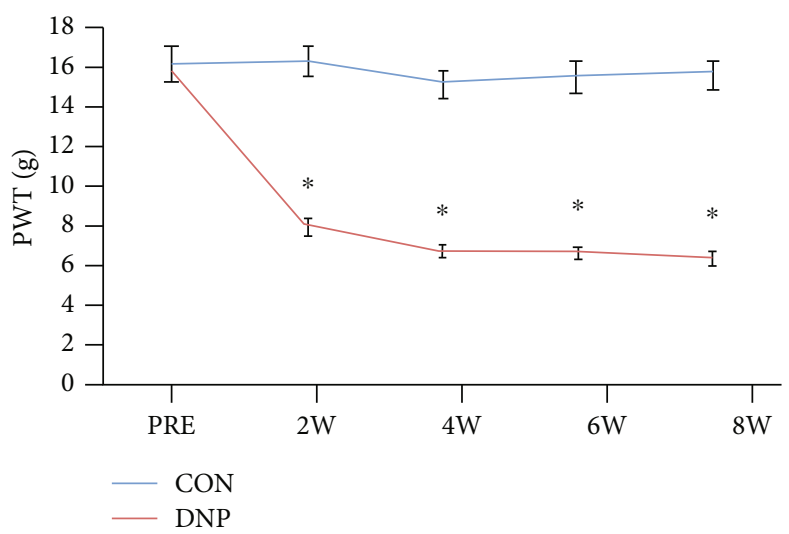

(h)

FIGURE 1: Changes of expression of TNFAIP3, ERK2, MAPK, and NF KB and pain threshold in Con and DNP groups. One-way ANOVA revealed significant variations across the groups $(P<0.05)$. The results of Western blot are as shown in $(\mathrm{a})$. (b)-(f) are the expression of TNFAIP3, ERK2, MAPK, and NF- $\kappa$ B, respectively. (g) and (h) are the results of PWL and PWT.

in the DNPsiRNA group vs. sham $\left({ }^{*} P<0.05\right)$; evidently lower TNFAIP3 in DNP-TNFAIP3 vs. DNP siRNA $\left({ }^{\#} P<0.05\right)$.

(6) Mechanical pain threshold and heat pain threshold changes and TNFAIP3 expression in the DNPsiRNA, DNP-TNFAIP3, and DNP INS groups

Figure 4 illustrates western blots of sham, DNPsiRNA, and DNP-TNFAIP3 groups and PWT (G) and PWL (S).

Overall, these data are suggestive of insulin loss or/and hyperglycemia but not STZ neuronal toxicity [10] as the most putative reasons attributed to predominant biochemical aberrations observed in this work.

\section{Discussion}

Diabetes is one of the focal and vital underlying disease and risk factors of ischemic stroke. Ischemic stroke patients with diabetes have more serious neurological deficits, slower recovery, worse intravenous thrombolysis effect, higher recurrence rate, and worse prognosis $[11,12]$. The mechanism remains to be comprehended completely with a vital involvement of inflammation displayed by studies. After cerebral ischemia-reperfusion, inflammatory cell activation is followed by the release of proinflammatory factors such as TNF- $\alpha$ and IL- $1 \beta$. Further adhesion, chemotaxis, and 


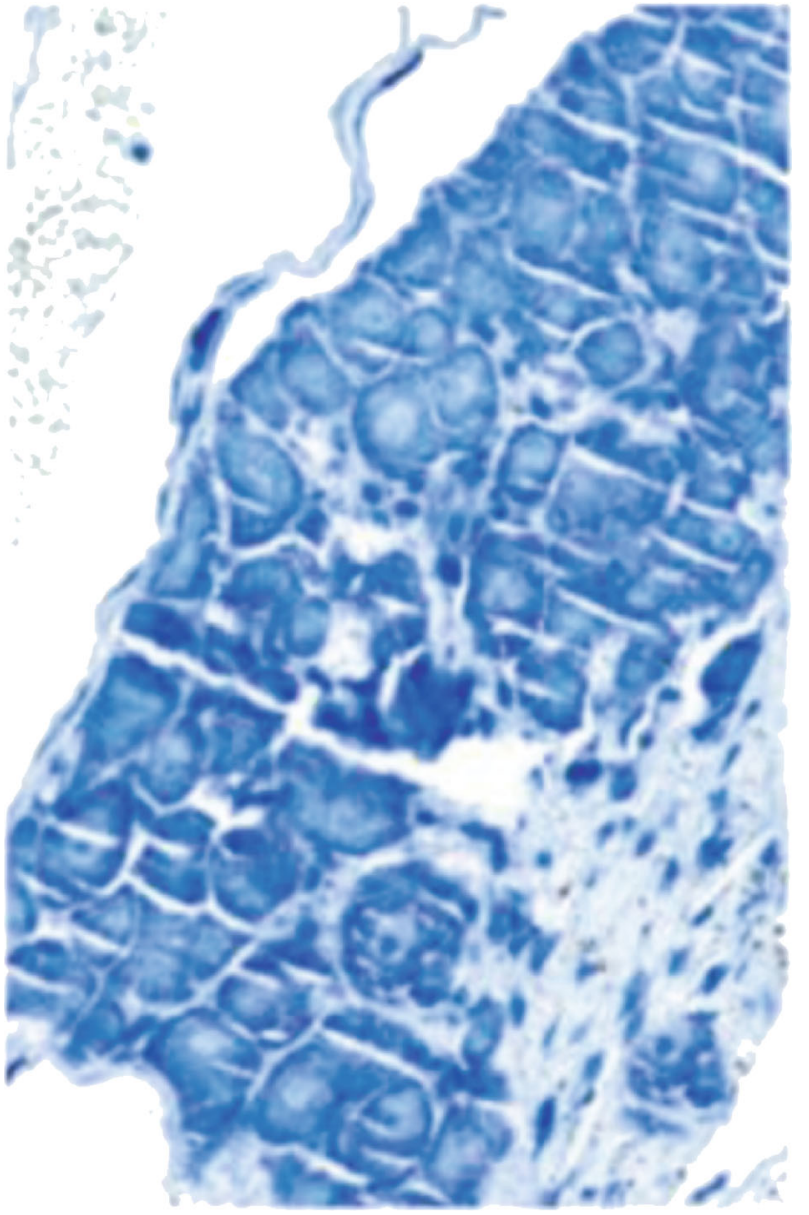

(a)

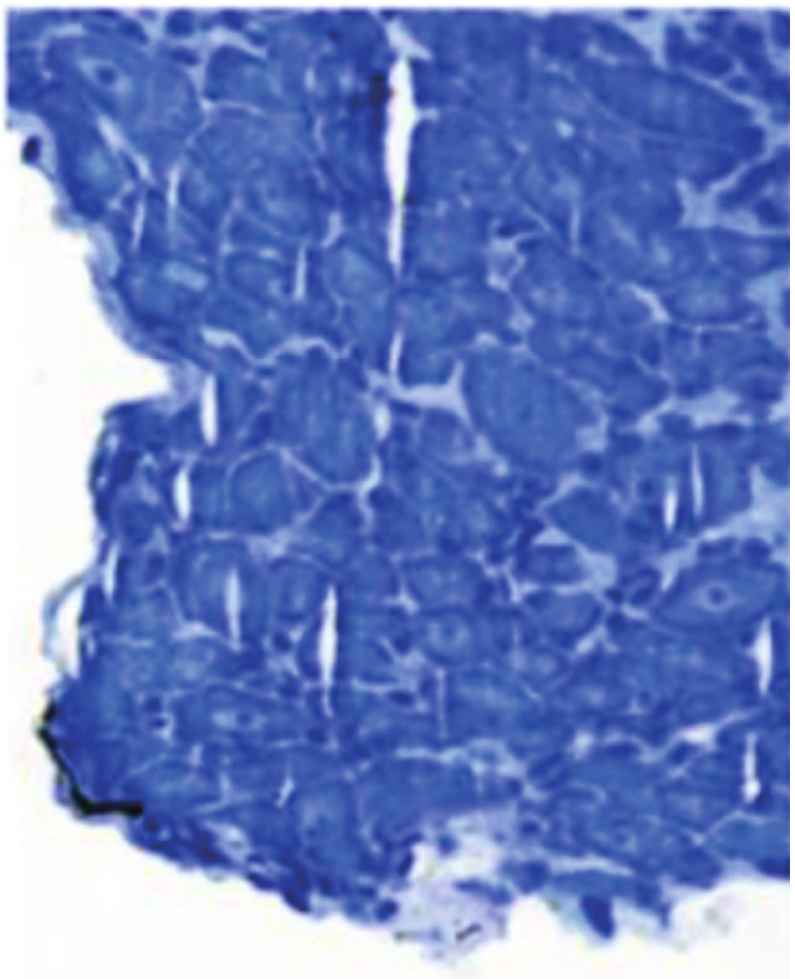

(b)

Figure 2: Continued. 


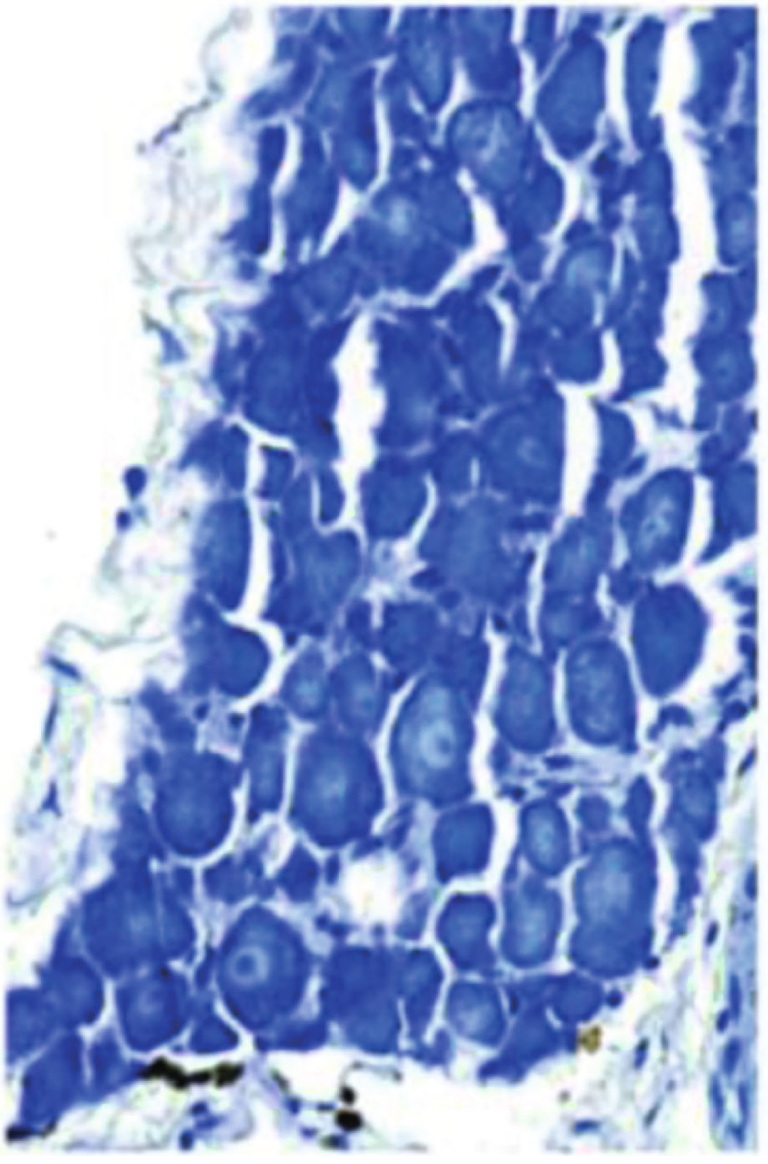

(c)

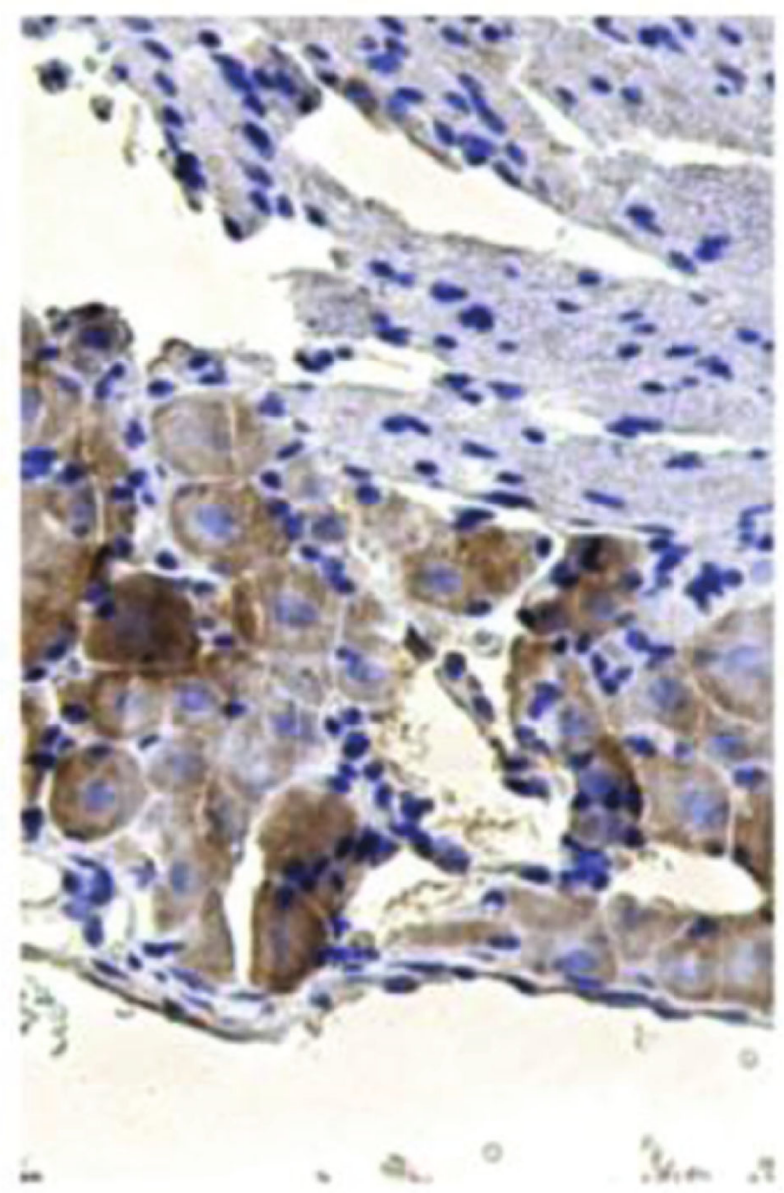

(d)

FIGURE 2: Continued. 


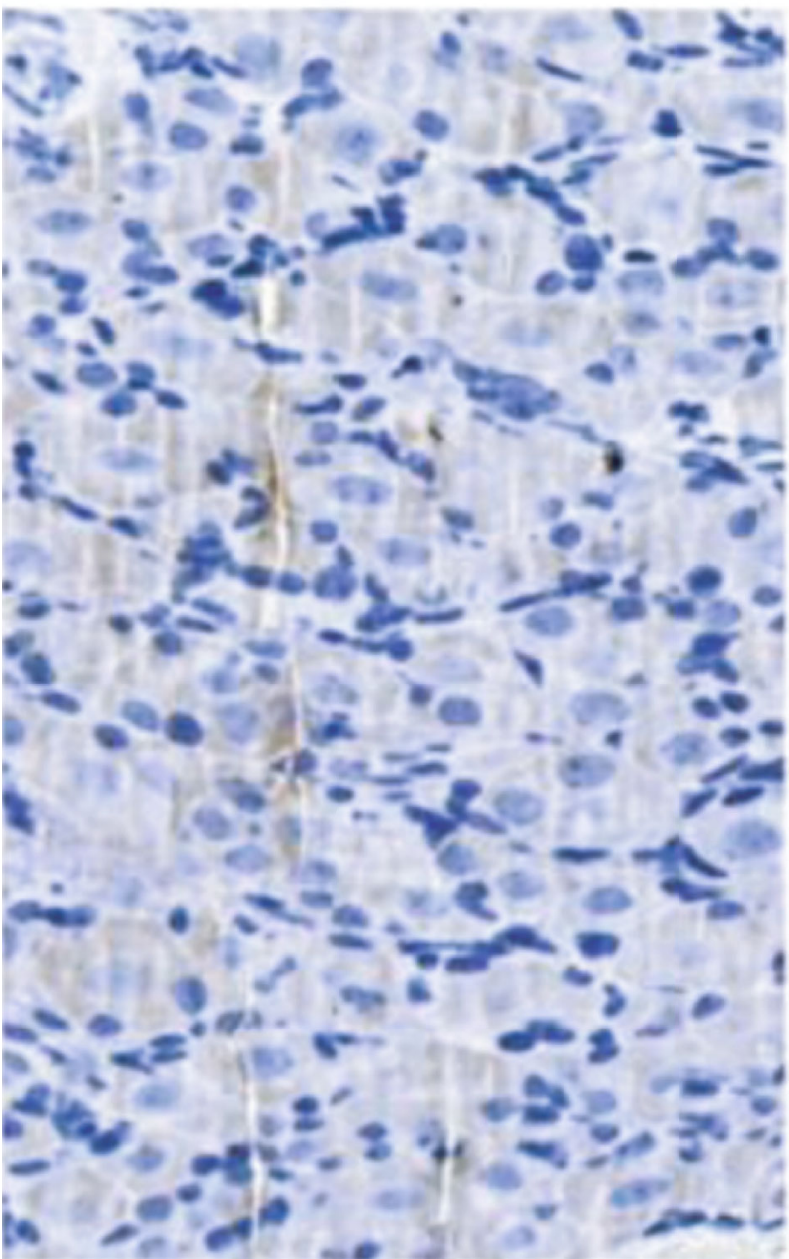

(e)

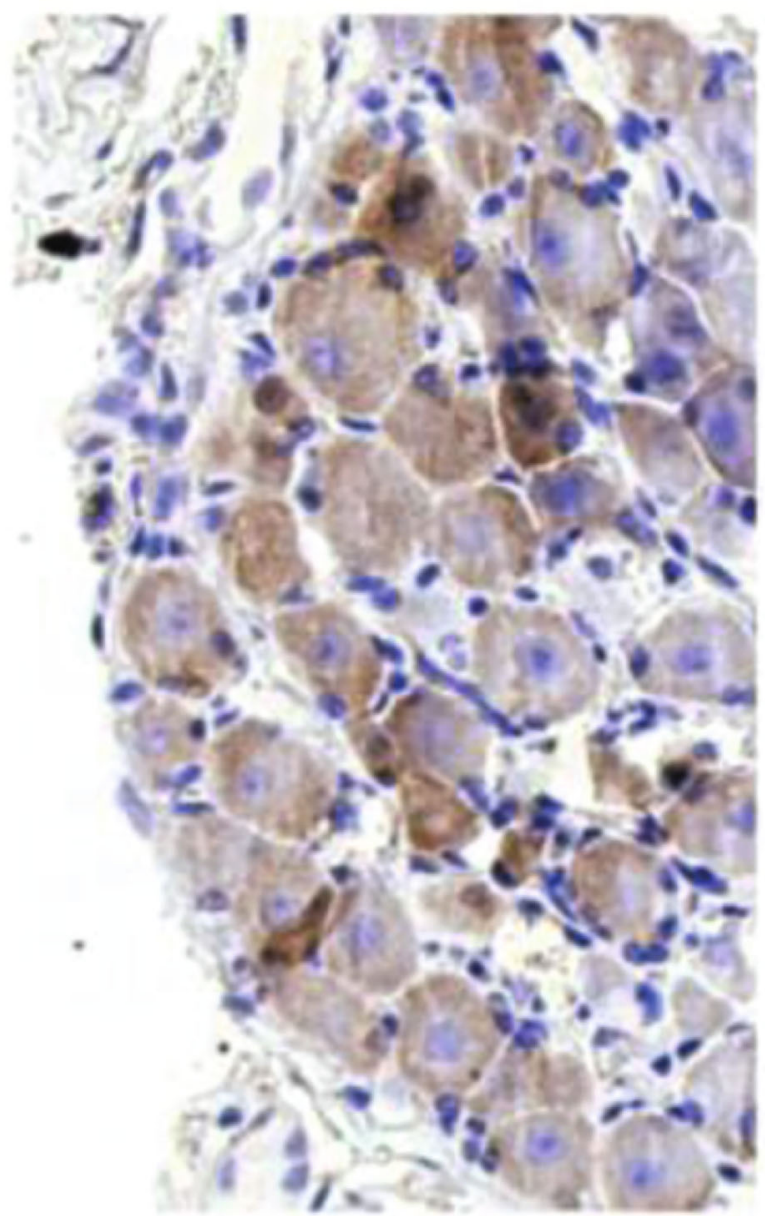

(f)

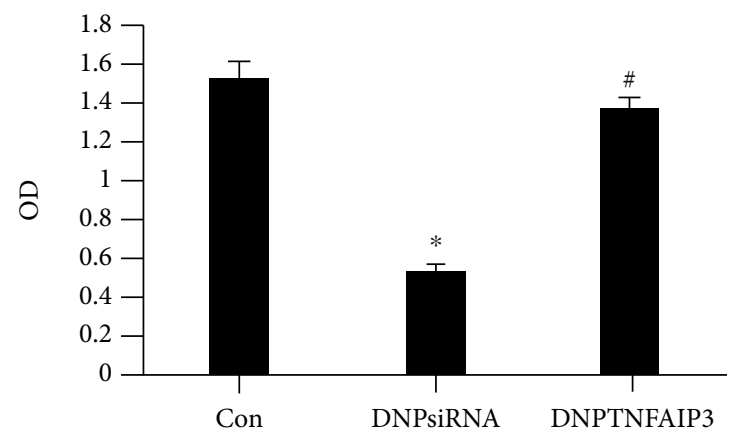

(g)

Figure 2: Morphological changes and TNFAIP3 expression in ganglion neurons in sham, DNPsiRNA, and DNP-TNFAIP3 groups. Oneway ANOVA statistically significant variations $(P<0.05)$. The results of toluidine blue staining are as shown in $(a)-(c)$ for sham, DNPsiRNA, and DNP-TNFAIP3 groups, respectively. The results of immunohistochemical staining are as shown in (d)-(f) for sham, DNPsiRNA, and DNP-TNFAIP3 groups, respectively. (g) is TNFAIP3 expression across the groups.

inflammatory cell activation result in a slew of factors as an inflammatory cascade to further damage the blood-brain barrier to cause ischemia-reperfusion injury via the NF- $\kappa \mathrm{B}$ pathway. This release of excitatory neurotransmitters directly or indirectly causes nerve damage $[13,14]$.

TNFAIP3, also known as zinc lipoprotein A20, is a member of the ubiquitin-editing enzyme family. It is both deubiquitinating and is an ubiquitin ligase. The vital involvement of this protein in regulating inflammatory signal transduction $[15,16]$ to negatively regulate the $N F-\kappa B$ pathway via feedback and inhibit NF- $\kappa$ B pathway activation via several inflammatory signals has been shown [17-20]. It can inhibit the transcription of downstream inflammatory factors and has a strong anti-inflammatory effect. Research 


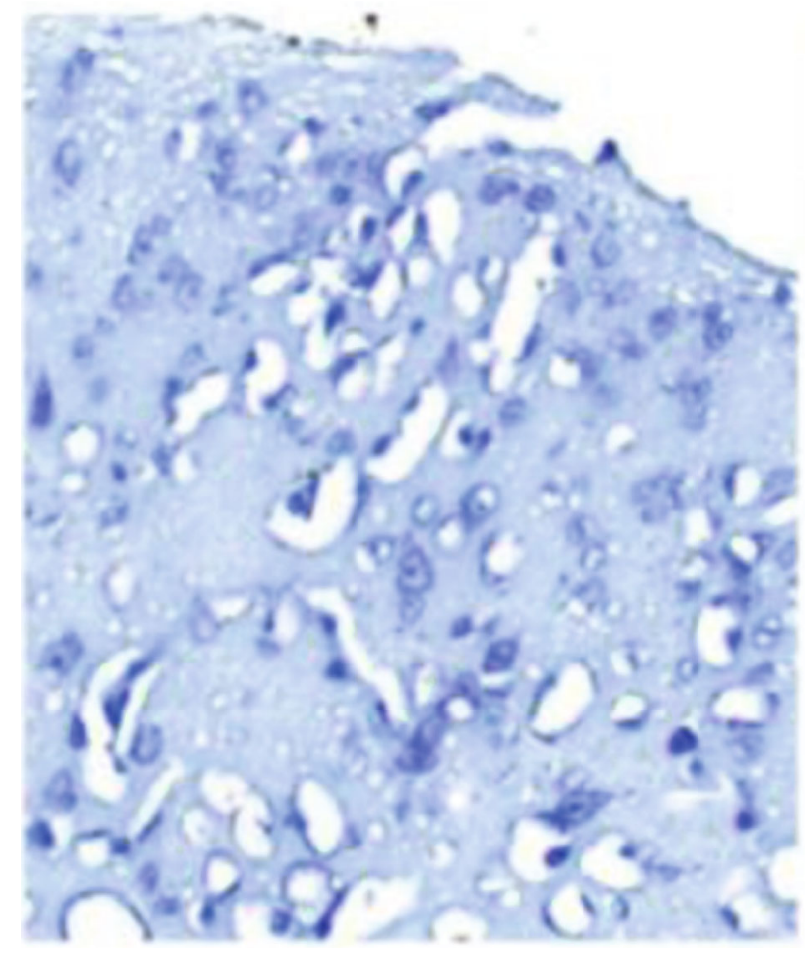

(a)

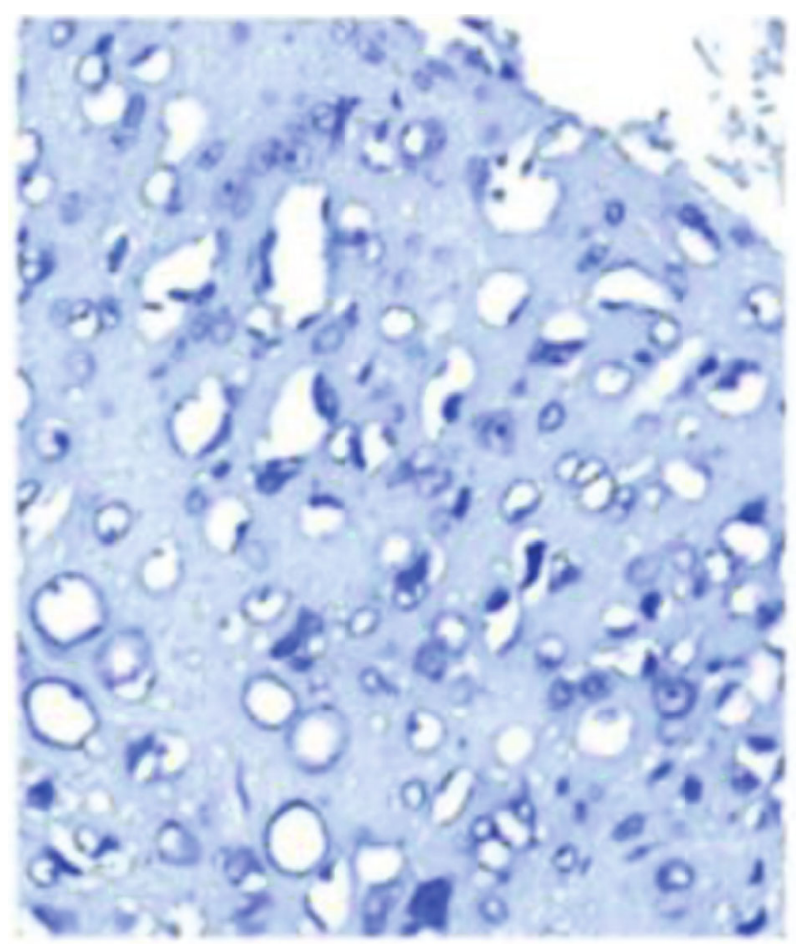

(c)

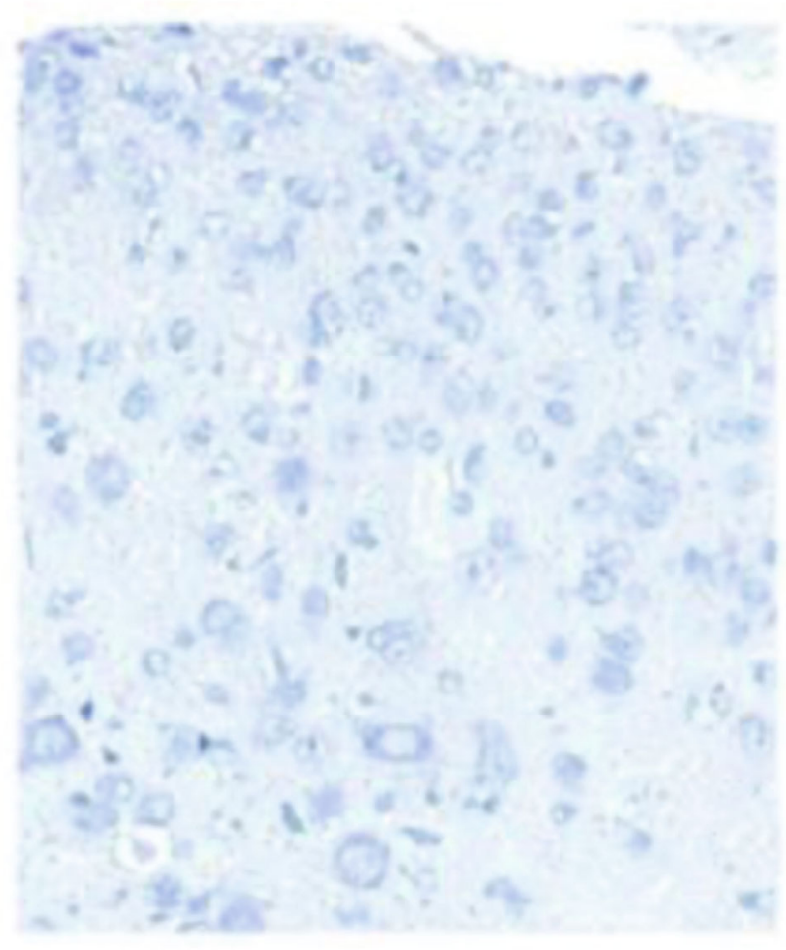

(b)

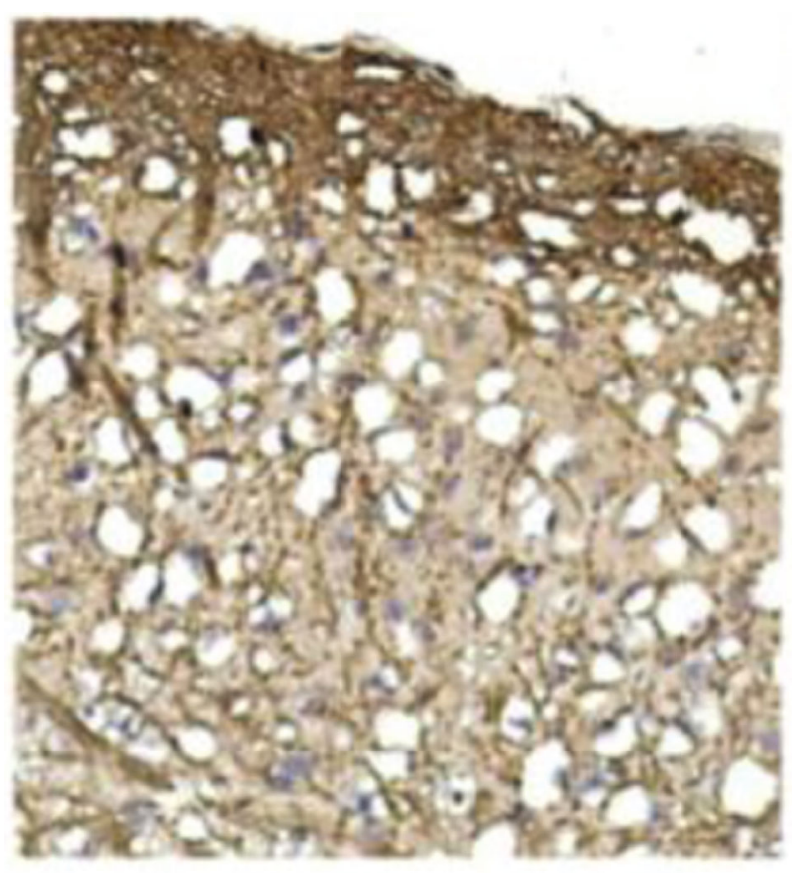

(d)

Figure 3: Continued. 


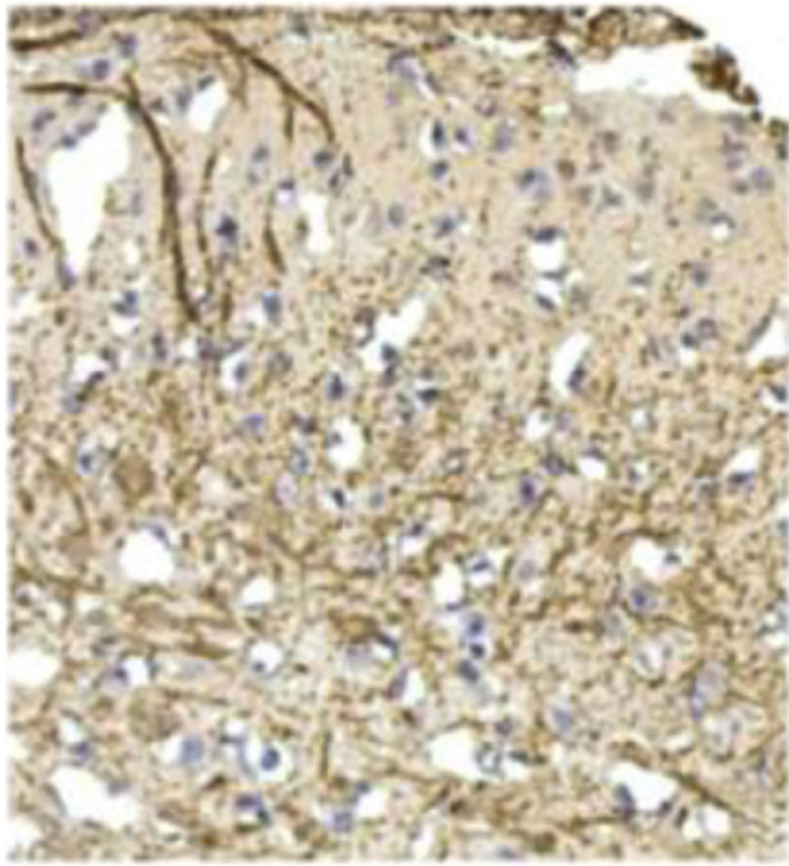

(e)

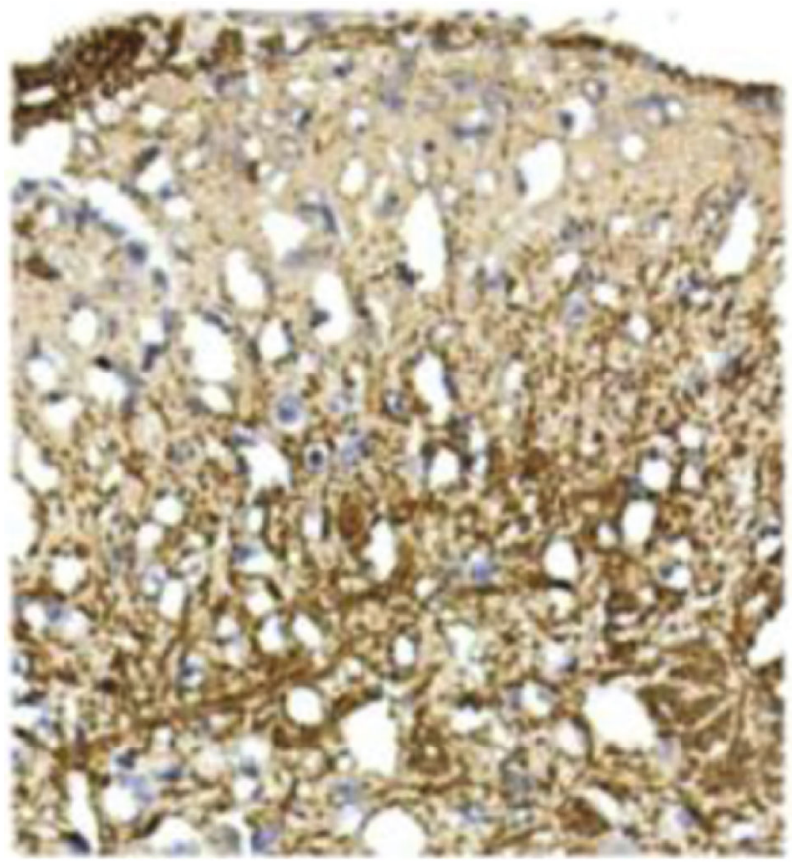

(f)

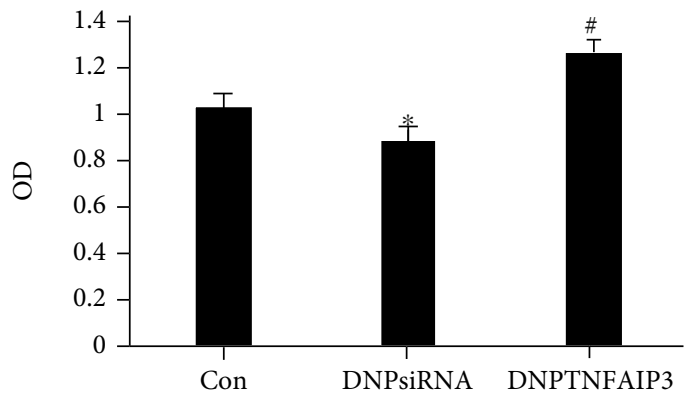

(g)

FIgURE 3: Morphological changes and TNFAIP3 expression in the posterior horn of the spinal cord in sham, DNPsiRNA, and DNPTNFAIP3 groups. One-way ANOVA revealed statistically significant variations $(P<0.05)$; the results of toluidine blue staining are as shown in (a)-(c): (a) is sham; (b) is DNPsiRNA group; (c) is DNP-TNFAIP3 group. The results of Immunohistochemical staining are as shown in (d)-(f): (d) is sham, (e) is DNPsiRNA group; (f) is DNP-TNFAIP3 group; (g) is TNFAIP3 expression across groups.

has pointed out to the alteration of this inflammation can lower the nerve damage caused by ischemia and reperfusion to thereby exert a protective effect on neurons. This inspired us to observe the analgesic effect of a lentivirus-based approach of high expression of TNFAIP3 via intrathecal injection into a diabetic neuropathic pain (DNP) mouse model. This was aimed at the impact of the significant upregulation of TNFAIP3 on DNP mice to further scrutinize the impact on DNP symptoms and unearth putative pain mechanisms. This work unveiled the crucial functioning of TNFAIP3 in DNP development.

Extracellular signal regulated kinase (ERK), a mitogen activated protein of the white kinase (MAPK) family, mediates the intracellular transduction of various signals [21, 22]. Elevated phosphorylation of this protein has been reported in different pain models. Given that murine hyperalgesia can be alleviated by blocking its upstream kinase, altering the activity can be linked to the transmission of deleterious stimulation and neurosensitivity. ERK activation is the key to signal transduction from the cell membrane surface receptor to the nucleus, with the involvement of upstream elements such as GTPase RAS, Raf-1, serine/threonine kinase, and bispecific kinase. ERK is crucially involved in the incidence and progression of pathological pain.

ERK includes two subtypes: extracellular signalregulated kinase-1 (erk-1) and extracellular signal-regulated kinase-2 (ERK-2). Gene knockout experiments show that ERK-2 can completely replace the function of erk-1, but erk-1 cannot replace the function of ERK-2 [23-25]. Therefore, this pathway can be scrutinized by blocking ERK-2. In addition, gene regulation also focally involves the role of nuclear factor kappa B (NFkB). Its activation can delay the apoptosis of neutrophils, prolong their life cycle, and increase their number, thus, activating and producing a large 


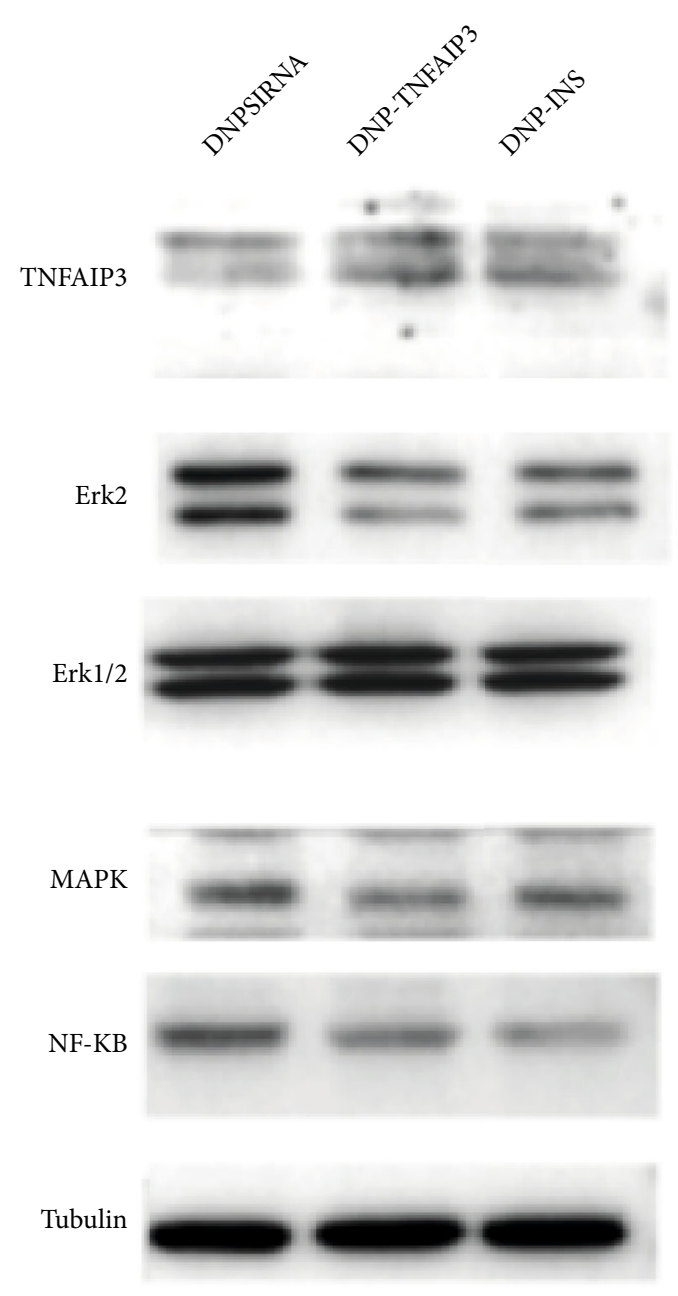

(a)

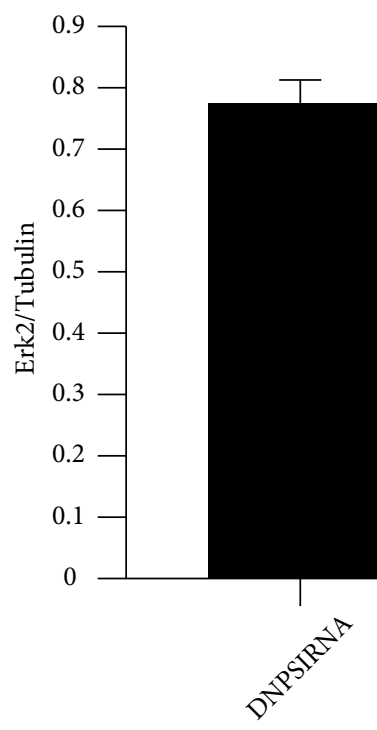

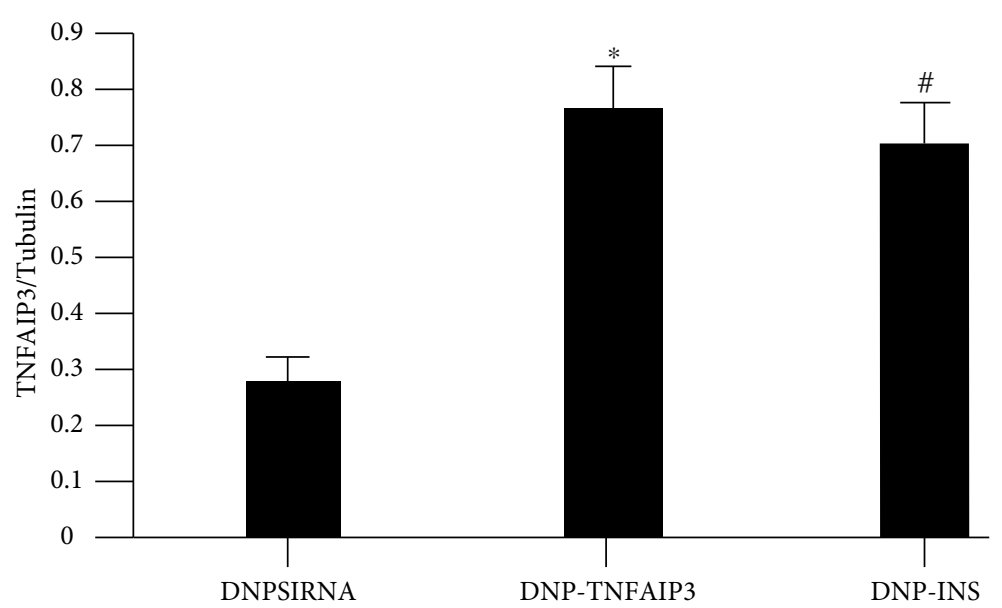

(b)

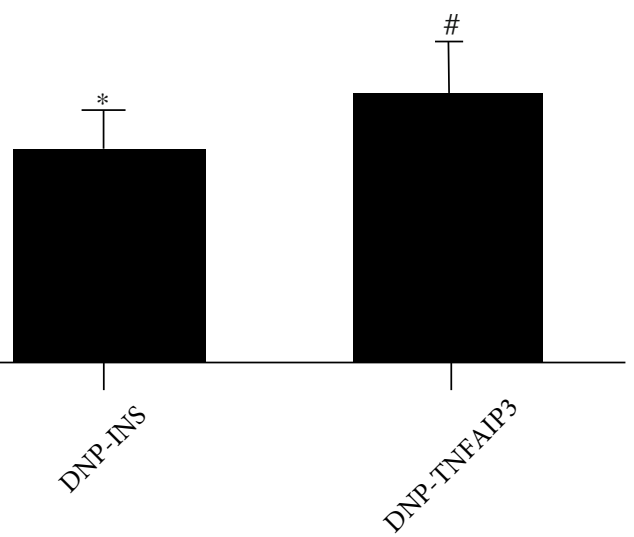

(c)

Figure 4: Continued. 


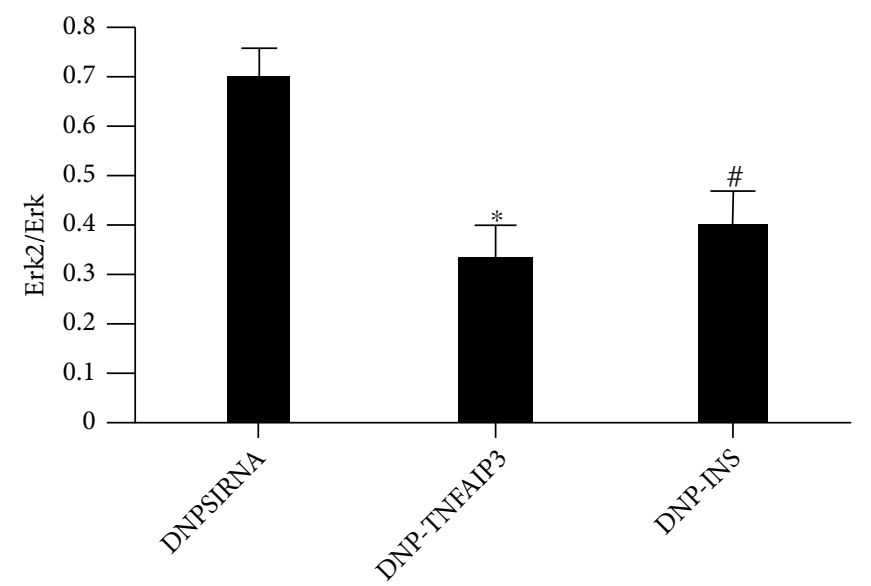

(d)

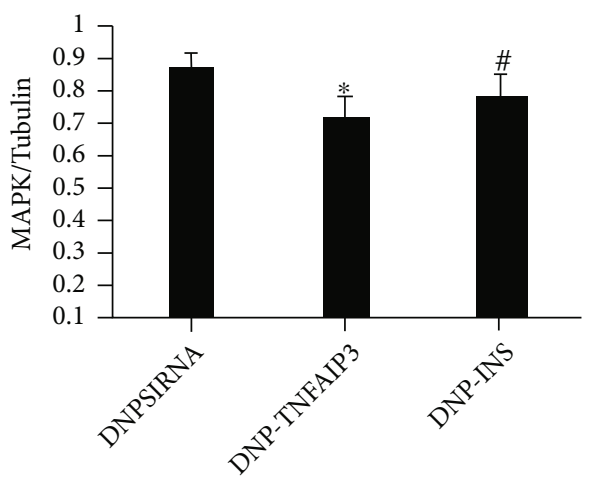

(e)

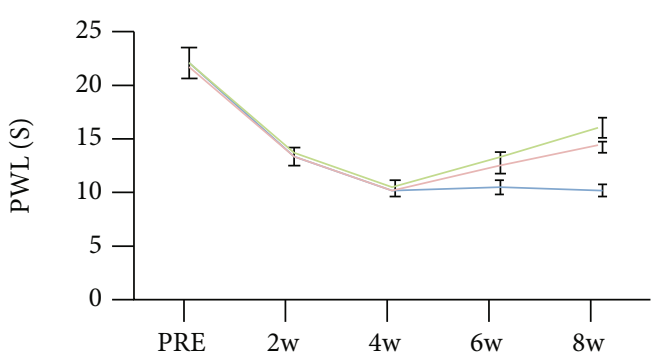

(g)

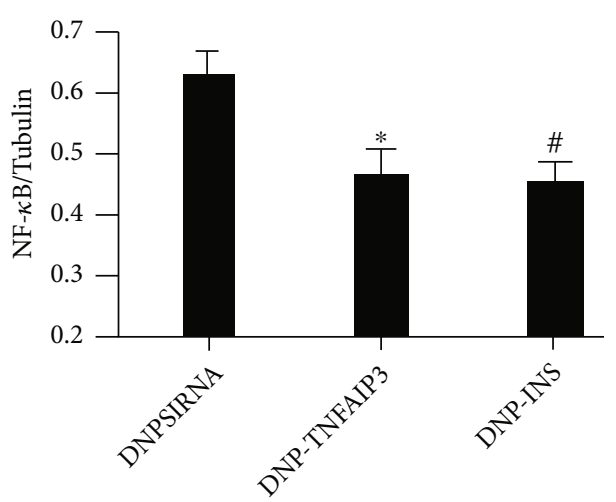

(f)

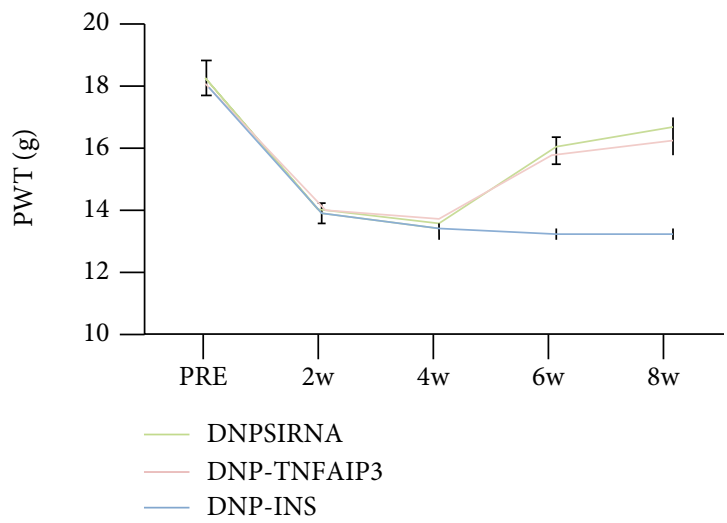

(h)

FIGURE 4: Expression changes of TNFAIP3, ERK2, MAPK, and NF KB in the posterior horn of spinal cord and pain threshold in sham, DNPsiRNA, and DNP-TNFAIP3 groups. One-way ANOVA revealed statistically significant alterations $(P<0.05)$. The results of Western blot are as shown in (a). (b)-(f) are TNFAIP3 expression, ERK2 expression, MAPK expression, and NF- $\kappa$ B expression. (g) and (h) are the results of PWL and PWT.

number of inflammatory mediators and free radicals. In the MAPK family, the ERK1/2-NFkB pathway is involved in cell growth, development, proliferation, differentiation, and apoptosis.

Scholars corroborated the augmentation of A20 by E. phaseoloides (ES) to inhibit the phosphorylation of ERK1/2 and p38 and target the inflammatory response in collagen-induced arthritis (CIA) rats [26]. Therefore, this work entailed ascertaining the effects of TNFAIP3 (referred to as "A20") on a murine DNP system. This inspired us to observe the analgesic effect via high expression of lentivirus-mediated TNFAIP3 by intrathecal injection in the animal model to explore its regulatory impacts, symptom relief, and mechanistic role in pain.

We demonstrate the impact of TNFAIP3 in murine DNP. In previous studies, we found that the expression of TNFAIP3 in a cerebral ischemia-reperfusion mouse model was significantly reduced. Two weeks after injection of LV- 
TNFAIP3, the neurological function score and cerebral infarction area were lowered indicative of the therapeutic effect of TNFAIP3 in mice. Western blot and immunohistochemical experiments highlighted evident restoration of TNFAIP3 protein after injection of LV-TNFAIP3 in the mouse system suggestive of the protective effect of TNFAIP3 on the brain tissue of the murine model of ischemiareperfusion. This led to the question of the putative protective impact of LV-TNFAIP3 on DNP mice. We started a follow-up experiment with LV-TNFAIP3 injection in the DNP mice to show increased thermal pain threshold and mechanical pain threshold 2 weeks after injection that is suggestive of the utility of LV-TNFAIP3 to treat DNP mice.

The next step was the assaying of the putative mechanism of specific treatment. The results revealed conspicuous lowering of ERK/NF-KB pathway proteins in the spinal cord and DRGs of the DNP mice following the upregulation of TNFAIP3. This is suggestive of an inhibited ERK/NF-KB following TNFAIP3 protein expression upregulation. The following step entailed exploring the neuroprotective mechanism of TNFAIP3 on the mice. Observations unveiled an evident suppression of the ERK/NF-KB pathway proteins suggestive of inhibition of this cascade via TNFAIP3 upregulation.

Our results show no evident alteration of the mechanical pain threshold and thermal pain threshold of the DNP group against the sham group, before (pre) modeling. The threshold of the DNP animals dipped at 2, 4, 6, and 8 weeks after modeling. At the 8th week after modeling, the spinal cord tissues were sampled for tests. Western blot and immunohistochemistry revealed a conspicuously lower TNFAIP3 expression in the DNP group against the sham.

In order to further study whether upregulation of TNFAIP3 expression affects the MAPK signaling pathway, further research was done. The pain threshold reported in this work displayed no evident change in DNP-TNFAIP3 before model making (PRE) and the DNP-INS groups as opposed to the DNPsiRNA group. Both thresholds displayed a conspicuous increase at 2, 4, 6, and 8 weeks after modeling for the DNP-TNFAIP3 and DNP-INS groups as against the DNPsiRNA group. As earlier mentioned, analyses of the 8th-week spinal cord samples revealed evidently higher TNFAIP3 expression in DNP-TNFAIP3 and the DNP-INS groups compared to the DNPsiRNA group.

\section{Conclusions}

In conclusion, according to the above analyses, the interaction between DNP and extracellular signal-regulated kinase signal transduction pathway is one of the key factors of pathogenesis. Intrathecal injection of adenovirus carrying multiple genes can significantly improve the pain threshold of mice with neuropathic pain to thus quell pain with efficacy. However, reports of the effect of lentivirus-based TNFAIP3 expression augmentation on diabetic neuropathic pain are scarce. Therefore, this project intends to further take up TNFAIP 3 as an effective target employing lentivirus as a vector to explore the effect and mechanism of upregulating TNFAIP3 on diabetic neuropathic pain.

\section{Data Availability}

All data relevant to the study are included in the article.

\section{Ethical Approval}

The study was approved by the animal ethics committee of Qiqihar Medical University China (approval no. QMUAECC-2021-199).

\section{Conflicts of Interest}

The author declares that there are no competing economic interests.

\section{Authors' Contributions}

Yuejing Wang and Yin Gao put forward the topic of the thesis, and Yuejing Wang and Yin Gao researched and analyzed the data and wrote the manuscript. Yang Liu and Jinhe Li analyze data and write manuscripts. Hongbo Yao helps discuss and review/edit the manuscript research and analyze data. Meng Zhang researches data and discusses and reviews/edits the manuscript. Jie Lian discusses and reviews/edits the manuscript research. Jinwei Chen researches data. Keshuang Zhang helps edit the manuscript. Danyang Liu designed the experiment, and Haiyan Zhang edited the manuscript.

\section{Acknowledgments}

This work was funded by Qiqihar Science and Technology Bureau (LHYD-202021), Qiqihar Medical Research Institute Project (QMSI2019M-11), and Qiqihar Science and Technology Bureau (SFGG-201759).

\section{References}

[1] I. Gilron, R. Baron, and T. Jensen, "Neuropathic pain: principles of diagnosis and treatment," Mayo Clinic Proceedings, vol. 90, no. 4, pp. 532-545, 2015.

[2] C. Gooch and D. Podwall, "The diabetic neuropathies," The Neurologist, vol. 10, no. 6, pp. 311-322, 2004.

[3] J. W. Russell and L. A. Zilliox, "Diabetic neuropathies," Continuum (Minneap Minn), vol. 20, pp. 1226-1240, 2014.

[4] C. A. Abbott, R. A. Malik, E. R. E. Van Ross, J. Kulkarni, and A. J. M. Boulton, "Prevalence and characteristics of painful diabetic neuropathy in a large community-based diabetic population in the U.K," Diabetes Care, vol. 34, no. 10, pp. 22202224, 2011.

[5] A. Peltier, S. A. Goutman, and B. C. Callaghan, "Painful diabetic neuropathy," BMJ, vol. 348, 2014.

[6] D. Ziegler, "Treatment of diabetic polyneuropathy: update 2006," Annals of the New York Academy of Sciences, vol. 1084, pp. 250-266, 2006.

[7] L. Zilliox and J. W. Russell, "Treatment of diabetic sensory polyneuropathy," Current Treatment Options in Neurology, vol. 13, no. 2, pp. 143-159, 2011.

[8] G. Y. Xu, G. Li, N. Liu, and L. Y. Huang, "Mechanisms underlying purinergic $\mathrm{P} 2 \mathrm{X} 3$ receptor-mediated mechanical allodynia induced in diabetic rats," Molecular Pain, vol. 7, 2011. 
[9] H. H. Zhang, J. Hu, Y. L. Zhou et al., "Promoted interaction of nuclear Factor- B with demethylated Cystathionine- Synthetase gene contributes to gastric hypersensitivity in diabetic rats," The Journal of Neuroscience, vol. 33, no. 21, pp. 9028-9038, 2013.

[10] M. Bishnoi, C. A. Bosgraaf, M. Abooj, L. Zhong, and L. S. Premkumar, "Streptozotocin-induced early thermal hyperalgesia is independent of glycemic state of rats: role of transient receptor potential vanilloid 1(TRPV1) and inflammatory mediators," Molecular Pain, vol. 7, 2011.

[11] J. Q. Han, C. L. Liu, Z. Y. Wang, L. Liu, L. Cheng, and Y. D. Fan, "Anti-inflammatory properties of lipoxin A4 protect against diabetes mellitus complicated by focal cerebral ischemia/reperfusion injury," Neural Regeneration Research, vol. 1, no. 4, pp. 636-640, 2016.

[12] H. Y. Zhao, T. Z. Zheng, X. H. Yang et al., "Cryptotanshinone attenuates oxygen-glucose deprivation/recovery-induced injury in an in vitro model of neurovascular unit," Frontiers in neurology, vol. 10, no. 4, 2019.

[13] V. Shukla, A. K. Shakya, M. A. Perez-Pinzon, and K. R. Dave, "Cerebral ischemic damage in diabetes: an inflammatory perspective," Joumal of Neuroinflammation, vol. 14, no. 1, 2017.

[14] M. M. Salman, P. Kitchen, M. N. Woodroofe et al., "Transcriptome analysis of gene expression provides new insights into the effect of mild therapeutic hypothermia on primary human cortical astrocytes cultured under hypoxia," Frontiers in Cellular Neuroscience, vol. 11, p. 386, 2017.

[15] M. Momose, T. Hirota, S. Kikuchi et al., "Associations of TNFAIP3 variants with susceptibility to psoriasis vulgaris and psoriasis arthritis in a Japanese population," Journal of Dermatological Science, vol. 100, no. 3, pp. 220-222, 2020.

[16] R. N. Duan, Q. Liu, J. X. Li et al., "Correction to: a de novo frameshift mutation in TNFAIP3 impairs A20 deubiquitination function to cause neuropsychiatric systemic lupus erythematosus," Journal of Clinical Immunology, vol. 40, pp. 10621063, 2020.

[17] P. Li, G. Zhao, F. F. Chen et al., "Rh-relaxin-2 attenuates degranulation of mast cells by inhibiting NF- $\kappa \mathrm{B}$ through PI3K-AKT/TNFAIP3 pathway in an experimental germinal matrix hemorrhage rat model," Journal of Neuroinflammation, vol. 17, no. 1, pp. 69-78, 2020.

[18] T. Kim, S. C. Bae, and C. Kang, "Synergistic activation of NF$\kappa \mathrm{B}$ by TNFAIP3 (A20) reduction and UBE2L3 (UBCH7) augment that synergistically elevate lupus risk," Arthritis Research \& Therapy, vol. 22, no. 3, pp. 913-921, 2020.

[19] F. Diomede, M. Zingariello, M. F. X. B. Cavalcanti et al., "MyD88/ERK/NFkB pathways and pro-inflammatory cytokines release in periodontal ligament stem cells stimulated by Porphyromonas gingivalis," European journal of histochemistry: EJH, vol. 61, no. 2, 2017.

[20] L. Shi, H. L. Tian, P. Wang et al., "Spaceflight and simulated microgravity suppresses macrophage development via altered RAS/ERK/NF $\kappa$ B and metabolic pathways," Cellular \& Molecular Immunology, vol. 18, no. 6, pp. 1489-1502, 2021.

[21] F. Shabani, M. Mahdavi, M. Imani, M. A. Hosseinpour-Feizi, and N. Gheibi, "Calprotectin (S100A8/S100A9)-induced cytotoxicity and apoptosis in human gastric cancer AGS cells: alteration in expression levels of Bax, Bcl-2, and ERK2," Human \& Experimental Toxicology, vol. 39, no. 8, pp. 10311045, 2020.

[22] J. H. Yang, L. M. Mao, E. S. Choe, and J. Q. Wang, "Synaptic ERK2 phosphorylates and regulates metabotropic glutamate receptor 1 in vitro and in neurons," Molecular Neurobiology, vol. 54, no. 9, pp. 7156-7170, 2017.

[23] Q. Yun, M. F. Jiang, J. Wang et al., "Overexpression Bax interacting factor-1 protects cortical neurons against cerebral ischemia-reperfusion injury through regulation of ERK1/2 pathway," Journal of the Neurological Sciences, vol. 357, no. 1-2, pp. 183-191, 2015.

[24] D. E. O'Brien, B. J. Alter, M. Satomoto et al., "ERK2 alone drives inflammatory pain but cooperates with ERK1 in sensory neuron survival," Journal of Neuroscience, vol. 35, no. 25, pp. 9491-9507, 2015.

[25] Z. Siprashvili, R. M. Shenoy, L. Elcavage, and P. Khavari, "130 small non-coding RNAs interact with ERK2 and effect MAPK/ERK pathway," Journal of Investigative Dermatology, vol. 140, no. 7, pp. S15-S15, 2020.

[26] H. Xiong, M. Luo, Y. K. Ju et al., “Triterpene saponins from Guo-gang-long attenuate collagen-induced arthritis via regulating A20 and inhibiting MAPK pathway," Journal of Ethnopharmacology, vol. 269, p. 113707, 2021. 